\title{
Estamos seguros? Reflexões sobre indicadores de avaliação da segurança alimentar e nutricional
}

\author{
Are we secure? Reflections on indicators \\ for evaluating food and \\ nutritional security
}

Elaine de AZEVEDO ${ }^{1}$

Maria Teresa Gomes de Oliveira RIBAS²

O conceito de segurança alimentar e nutricional, construído coletivamente no cenário brasileiro, apresenta-se como um elemento catalizador de importantes reflexões na área de Nutrição Social. Este texto tem como objetivo refletir sobre as formas e os indicadores de avaliação em segurança alimentar e nutricional no Brasil. Esta breve reflexão se debruça sobre os desafios dos especialistas e pesquisadores da área em questão para abarcar seus princípios interdisciplinares; além disso, espera motivar o interesse pela elaboração de novos indicadores de avaliação e de metodologias e instrumentos de coleta de informação mais abrangentes e sistêmicos. Julga-se importante promover a reflexão sobre as limitações das escalas de mensuração da (in)segurança alimentar e nutricional em apontar e abarcar as complementaridades entre a vivência dessa insegurança e os processos e agentes de produção do fenômeno. Ressalta-se como desejável a inclusão da leitura do processo saúde-doença e o cuidado das morbidades resultantes da insegurança alimentar e nutricional como questões a serem exploradas em futuros estudos dessa temática.

Palavras-chave: Alimentos naturais. Saúde pública. Segurança alimentar e nutricional.

A B S T R A C T

The concept of food security, collectively constructed in the Brazilian scenario, is considered an important element to promote reflections in the area of Social Nutrition. This article aims to reflect on the evaluation indicators for food and nutrition security in Brazil. This brief reflection focuses on the challenges of experts and

\footnotetext{
1 Universidade Federal do Espírito Santo, Centro de Ciências Humanas e Naturais, Departamento de Ciências Sociais. Av. Fernando Ferrari, 514, Goiabeiras, 29075-910, Vitória, ES, Brasil. Correspondência para/Correspondence to: E AZEVEDO. E-mail: <elainepeled@gmail.com>.

2 Pontifícia Universidade Católica do Paraná, Escola de Saúde e Biociências, Curso de Nutrição. Curitiba, PR, Brasil.
} 
researchers in food and nutritional security to embrace its interdisciplinary principles. One expects to motivate the interest in developing new indicators and evaluation methodologies and more comprehensive and systemic instruments to collect information about food insecurity. It is deemed important to promote reflection on the limitations of the scales of measurement of food and nutrition (in)security in pointing and covering the complementarities between the experience of such insecurity and the processes and agents that produce this phenomenon. Issues to be explored in future studies are the inclusion of understanding the health-disease process and the care of morbidity resulting from food and nutrition insecurity.

Keywords: Health foods. Public health. Food security and nutrition.

\section{N T R O D U Ç Ã O}

A ideia deste trabalho é compartilhar reflexões sobre as formas de analisar e mensurar o complexo conceito de Segurança Alimentar e Nutricional (SAN). Esse termo foi construído coletivamente no cenário nacional brasileiro a partir do processo de reativação do Conselho de Segurança Alimentar e Nutricional (Consea), em 2003'.

A segurança alimentar e nutricional apresenta-se como um elemento provocador de importantes ações e discussões interdisciplinares; é a diretriz da Política Nacional de Segurança Alimentar e Nutricional (PNSAN)2 , homologada em 2010; tem provocado mudanças estruturais em políticas públicas de desenvolvimento social, de agricultura, de alimentação e de nutrição e instigado propostas de reestruturação da área de Nutrição Social. Além disso, a construção da segurança alimentar e nutricional influenciou o conceito de alimento saudável contemporâneo, o qual superou as premissas reducionistas modernas baseadas em prescrição quantitativa de nutrientes, e incorporou o termo 'adequado' e os elementos culturais e socioambientais ${ }^{3,4}$.

Identificar experiências e campos de reflexão sobre a promoção da segurança alimentar e nutricional tornou-se o foco de importantes editais de conselhos e fundações nacionais de apoio à pesquisa. Com isso, pode-se considerar que a área técnica da Nutrição, enquanto espaço especializado da saúde na administração pública, não carrega mais o pesado fardo de promover setorialmente a alimentação saudável. Ou seja, é possível vislumbrar que a perspectiva conceitual da segurança alimentar e nutricional potencializou a dissolução de barreiras disciplinares tornando mais porosas e articuladas as áreas de produção e consumo de alimentos, ciências humanas e sociais, meio ambiente e desenvolvimento rural. Esse conceito consolida a ideia da realização do direito universal ao acesso regular e permanente a uma alimentação saudável e adequada que seja cultural, social, econômica e ambientalmente sustentável ${ }^{5}$.

A alimentação saudável e adequada é concebida tendo como pauta o referencial tradicional e local de produção e consumo. Objetiva fomentar a soberania alimentar e promover sistemas de produção familiar e sustentável, com o mínimo de impactos socioambientais e de desigualdades relacionadas ao acesso à terra. Deve atender aos princípios de variedade, equilíbrio, moderação e prazer e considerar as dimensões de gênero e etnia e priorizar alimentos livres de contaminantes físicos, químicos, biológicos e de produtos transgênicos. Clama por respeitar aspectos biológicos e sociais dos indivíduos, considerando as especificidades de cada fase do curso da vida e também suas necessidades especiais. Isso significa que comer e ser obeso, diabético, celíaco, alérgico, estéril ou ter câncer também significa viver - ou morrer -, sob condições de insegurança alimentar. Diante dessas premissas, é possível perceber que alcançar a segurança alimentar e nutricional é um desafio e um anseio ético de fomentar mudanças estruturais na sociedade brasileira e no sistema agroalimentar dominante, bem como de minimizar as inequidades étnico-raciais, de gêneros, sociais e geracionais.

O conceito transcende o aspecto quantitativo e biologicista inerente à segurança sanitária e assume a ideia de qualidade de alimentos isentos de contaminantes, não somente biológicos e 
genéticos, mas também físicos e químicos, os quais sejam ajustados à realidade cultural da região onde são produzidos. Preocupa-se também com a segurança e a dignidade dos agentes sociais envolvidos na produção do alimento.

Uma publicação do Consea Nacional ${ }^{6}$ estabelece as premissas orientadoras de uma Política de Segurança Alimentar e Nutricional para o País e não deixa de considerar dois segmentos de atores relacionados às situações de insegurança alimentar e nutricional: os sujeitos que vivenciam o problema e os agentes de produção do problema. Esses últimos, considerados aqueles que promovem a produção ou produzem alimentos a partir de modelos predatórios ao meio ambiente, às relações econômicas e sociais ou a partir de tecnologias não comprovadamente seguras; que promovem a prática ou praticam preços abusivos em relação aos alimentos e bens essenciais; que negam informação ou ignoram o dever de informar a composição nutricional e todos os componentes presentes nos produtos alimentares; e que promovem a imposição de padrões alimentares sem respeito à diversidade cultural das sociedades.

Isso significa que ter fome, não ter o que comer regularmente, não ter recursos para comprar alimentos, ser desnutrido ou pobre são apenas algumas das situações tradicionalmente identificadas com a vivência do não alcance da segurança alimentar e nutricional. Na realidade, esses elementos foram tradicionalmente determinantes para legitimar uma situação insegura de acesso aos alimentos, mas o novo conceito alargou-se consideravelmente.

\section{Avaliando a (in)segurança alimentar e nutricional}

Apesar da laboriosa tarefa de construção social de um conceito ampliado de segurança alimentar e nutricional, serão discutidos aqui os elementos que ainda predominam nos instrumentos de avaliação da mesma. A Escala Brasileira de Insegurança Alimentar (EBIA), utilizada na Pesquisa Nacional por Amostras de Domicílio
(PNAD) ${ }^{7}$, é composta de 15 itens que se debruçam sobre as dimensões quantitativa e econômica da insegurança alimentar: porções insuficientes, finitude ou restrição quantitativa da comida em casa, sentimento de fome, falta de dinheiro para comprar comida, poucas opções de alimentos por falta de recursos financeiros para compra, etc. Os resultados dessa escala são inseridos em categorias quantitativas: insegurança alimentar leve, moderada ou grave ${ }^{8}$.

Pode-se considerar o instrumento da EBIA como um roteiro, diferente de um questionário, com itens delineadores de uma entrevista que, apesar da resposta finalista dicotômica, visa captar a percepção ou o reconhecimento dos sujeitos sobre a vivência da insegurança alimentar e nutricional em diversos patamares de intensidade. Ou seja, a configuração do instrumento, que conduz a uma focalização gradativa da (in)segurança alimentar e nutricional como fenômeno concreto, permite, ao longo da elaboração das perguntas, um posicionamento do entrevistado e/ou de seu núcleo familiar em relação ao problema. Sua contribuição, sob a ótica de aplicação extensiva populacional, é viabilizar a produção de um indicador quantificável que não desvalorize as subjetividades da experiência da segurança ou insegurança alimentar e nutricional. Dessa forma, cada pergunta elaborada é, em sua essência, uma narrativa dos sujeitos, expondo os encadeamentos singulares da experiência vivida acerca da sua segurança ou insegurança alimentar e nutricional, a qual desencadeia o processo decisório da resposta. Nesse processo, reside a riqueza subliminar desse instrumento que não deve ser negligenciado.

Ainda em termos concretos, a aplicação da EBIA conseguiu inserir a SAN no rol da produção sistemática de informação, o que permite avaliar tendências, impactos e pontos de inversão dessa fenômeno em nível populacional. O que representa um ganho político, portanto, quando essa temática passa a ser evidenciada em um campo 'oficial' de análise. Além disso, ao identificar os patamares de medida da vulnerabilidade da 
condição alimentar e nutricional, também foi viabilizada sua articulação com outros indicadores relevantes que podem ampliar o delineamento mais efetivo da (in)segurança alimentar e nutricional - como o estudo do Instituto Brasileiro de Análises Sociais e Econômicas ${ }^{9}$ que articula os resultados da EBIA à inequidade territorial das regiões brasileiras, à renda per capita das famílias e aos quesitos raça/cor, escolaridade, situação de trabalho e acesso ao trabalho formal do titular.

Esse tem sido o instrumento eleito para uma avaliação mais aproximada da vulnerabilidade em termos de alimentação e nutrição de núcleos familiares, o que representa um avanço no que diz respeito às metodologias tradicionais, de viés economicista, focadas na disponibilidade geral de alimentos por população. O valor central desse instrumento pode ser identificado em seu potencial de captar percepções sobre o vivido.

Além dessa escala psicométrica de acesso familiar aos alimentos, Pérez-Escamilla \& Segall-Corrêa apontam outros métodos para avaliação da insegurança alimentar e nutricional que, para as autoras, focam pontualmente sobre uma matemática de disponibilidade geral de nutrientes ou que se debruçam sobre o consumo alimentar (perspectivas quantitativas que a EBIA se propõe a superar): o método de cálculo da disponibilidade calórica diária per capita da Food and Agriculture Organization; o cálculo do rendimento mínimo para consumo alimentar e não alimentar; o cálculo do consumo alimentar (como os recordatórios quantitativos das últimas 24 horas, frequência de consumo alimentar ou a quantiûcação dos gastos familiares com aquisição de alimentos); a avaliação do consumo dietético individual e a antropometria.

Ressalta-se que, apesar de ser inserida como quantitativa por Pérez-Escamilla \& Segall-Corrêa ${ }^{8}$, a antropometria é uma complexa forma de sintetizar processos sociais que se expressam como desfecho biológico na condição ou no estado nutricional do indivíduo. Kepple et al. ${ }^{10}$ enfatizam a importância dessas escalas de medidas (junto a outros indicadores de disponibilidades de alimentos) como recurso metodológico eficaz para elucidar determinantes de insegurança alimentar e nutricional, bem como para apontar sua magnitude e os efeitos sobre a saúde e nutrição dos indivíduos.

\section{As limitações das escalas de Insegurança e Segurança Alimentar e Nutricional}

Em dados estimados pela PNAD de 2013, em $76,7 \%$ da população urbana pesquisada nos domicílios particulares do Brasil residiam pessoas em situação de segurança alimentar, ou seja, seus moradores, nos 90 dias prévios à data da entrevista, tiveram acesso aos alimentos em quantidade e qualidade adequadas e não se sentiam na iminência de sofrer qualquer restrição no futuro próximo. Isso significa que esses indivíduos alcançaram a condição de estabilidade que implica no grau de perenidade da utilização, acesso e disponibilidade dos alimentos. A mesma pesquisa revela que o patamar de segurança alimentar cai para $59,9 \%$, em domicílios rurais, e atinge $36,7 \%$, onde o rendimento mensal domiciliar per capita é de até $1 / 4$ do salário mínimo7.

Entretanto, apesar do detalhamento dos dados e dos avanços no esforço de explicitar a insegurança alimentar e nutricional, a utilização da escala brasileira e dos outros indicadores para detectar seus níveis não identifica o desequilíbrio e a erosão cultural da dieta e dos sistemas alimentares ou a contaminação química e genética dos alimentos como elementos de insegurança. Burlandy \& Salles-Costa ${ }^{11}$ afirmam que a concepção que subsidia da EBIA refere-se mais à escassez alimentar no nível domiciliar e menos à segurança alimentar e nutricional como proposta em sua concepção multidimensional. Além da privação de alimentos, as ideias de qualidade e variedade inseridas na escala remetem a aspectos qualitativos na percepção da família forjada em seus sistemas de valores culturais e simbólicos sobre a comida, que não necessariamente repre- 
sentam as dimensões técnicas implícitas no conceito de segurança alimentar e nutricional.

Quando alguns estudos conseguem transcender essa dimensão quantitativa, mencionam a contaminação biológica e o perigo dos microrganismos patogênicos. O esforço em inserir questões qualitativas na EBIA ainda não resolveu a prevalência da perspectiva quantitativa e sanitária do conceito de segurança alimentar dentro da dimensão de falta de acesso ou carência de alimentos, referências centrais da nutrição moderna.

Percebe-se a preocupação de pesquisadores $^{12}$ os quais utilizam estudos prévios ${ }^{13,14}$ para enfatizar a incapacidade da EBIA de apreender a (in)segurança alimentar e nutricional do meio rural, remetendo a questões culturais que circundam a discussão de SAN:

Em relação ao meio rural, ainda, a escala não analisa a possibilidade de produção agrícola para autoconsumo e seus modos coletivos de produção (extrativismo, faxinais, fundos de pasto, assentamentos, etc.) ou práticas não mercantis de acesso aos alimentos (reciprocidade, trocas, dádivas). Questões sobre posse de terra, acesso à água ou a insumos agrícolas, origem dos alimentos, contaminação microbiológica ou com metais pesados e pesticidas não são capturadas, assim como a situação da safra atual no momento da aplicação da escala (p.53) $)^{12}$.

Entretanto, nos estudos que abordam especificamente a avaliação da insegurança alimentar nutricional em populações que têm por base a aplicação da EBIA, não são mencionados os elementos estruturais que colocam os indivíduos em situação de insegurança. O que se pretende enfatizar com essa afirmação é a dificuldade de assumir as complexas diretrizes e princípios implícitos no conceito quando o estudo se propõe a mensurar insegurança alimentar e nutricional, considerando sua complexa trama de dimensões que devem estar simultaneamente articuladas para que o direito humano à alimentação adequada se realize de modo efetivo.

\section{Uma problemática não revelada nas escalas de Insegurança e Segurança Alimentar e Nutricional}

O Brasil é o primeiro consumidor de agrotóxicos do mundo e, segundo dados do Dossiê da Associação Brasileira de Saúde Coletiva ${ }^{15}$, 70\% dos alimentos in natura aqui consumidos estão contaminados intencionalmente por produtos os quais já são proibidos em outros países, além de serem inadequados às culturas onde são utilizados. O Programa Nacional para a Redução de Uso de Agrotóxicos (Pronara) que propõe a regulamentação dos agroquímicos está ameaçado de não ser implementado pelo Ministério da Agricultura. Entre os 50 ingredientes ativos mais usados no Brasil, quase a metade - 22 -, são proibidos na União Europeia ${ }^{15}$. Nesse resultado grãos ou produtos de origem animal não foram analisados pelo Programa de Análise de Resíduos de Agrotóxicos da Agência Nacional de Vigilância Sanitária (Anvisa) ${ }^{16}$. Também estão fora dessa conta outros contaminantes, como adubos e aditivos sintéticos, drogas veterinárias, produtos da irradiação e também a análise de riscos dos alimentos com organismos geneticamente modificados.

Ainda existe carência de informações sistematicamente coletadas e confiáveis que permitam uma análise quantitativa precisa do consumo de alimentos industrializados no Brasil. Sabe-se, porém, que o mesmo cresceu $218 \%$ entre 1974 e 2002 e que o aumento do seu volume passou de 1,7 kg per capita em 1974 para 5,4 kg em 2002 ${ }^{17}$.

Não se pode deixar de mencionar a situação de vulnerabilidade dos agricultores familiares - os quais recebem apenas $25 \%$ de incentivo à produção apesar de serem os agentes de produção de quase $80 \%$ da alimentação culturalmente referenciada dos brasileiros. Como inserir de forma mais efetiva na avaliação de segurança alimentar e nutricional a preocupação com o fato de que grande parte da dieta dos brasileiros é composta por alimentos desterritorializados, ou seja, desconectados geograficamente e cultural- 
mente do local onde foram produzidos? Além disso, não é possível ignorar a ameaça de extinção de 800 tipos de alimentos locais ${ }^{18}$, os quais, certamente, não são de interesse do setor econômico do agronegócio de exportação.

Motivados por essas inquietantes constatações é importante perguntar: estaria efetivamente segura essa parcela majoritária da população brasileira que, segundo estudos sobre vulnerabilidade à segurança alimentar e nutricional, demonstra ter acesso ao alimento? Questiona-se se a alimentação desses indivíduos tem sido capaz de prevenir disfunções compiladas em estudo de Azevedo \& Rigon ${ }^{19}$. São enfermidades relacionadas ao uso de agrotóxicos, fertilizantes e aditivos sintéticos, drogas veterinárias e produtos provenientes da irradiação, como alguns tipos de câncer, imunodepressão, doença de Parkinson, depressão e outros distúrbios neurológicos, autismo, aborto, esterilidade, problemas congênitos e respiratórios, alergias, algumas formas de anemia e resistência a antibióticos.

$\mathrm{E}$, para além desse quadro, outras questões devem ser consideradas nesse contexto. A dieta prevalecente é composta por alimentos industrializados, ricos em açúcar, sal, gorduras trans e aditivos sintéticos. Por fim, os rumores que circulam na mídia alertando sobre a origem, a contaminação ou a fraude de diferentes alimentos também não devem tranquilizar especialistas e consumidores leigos. Esses alertas veiculam dados que, mesmo carentes de comprovação científica, provocam inquietude e ansiedade, sentimentos que podem ser caracterizados como insegurança para o consumidor, a partir da incorporação social e cultural da ideia de 'risco'. Se essas informações veiculadas nos sites a seguir mobilizarem estudos futuros, já vale a menção às mesmas (Quadro 1).

No mínimo, esses alertas levam a perguntar se a população está realmente saudável consumindo alimentos que, com o apoio do sistema econômico-industrial e da propaganda massiva, constroem e propagam valores de segurança e até de funcionalidade. Alimentos provenientes de um sistema que torna a sociedade dependente de grãos comercializados e produzidos por apenas cinco empresas multinacionais, que destrói as florestas e a biodiversidade, que interfere no clima, que inviabiliza terras agriculturáveis e que polui rios lagos e mares, o ar e o solo? ${ }^{20}$. É possível encontrar indivíduos saudáveis (e seguros) vivendo em um meio ambiente insalubre e sob condições vulneráveis de soberania alimentar? É saudável um alimento cuja produção promove exclusão social, trabalho escravo e poluição ambiental?

É preciso, ainda, refletir em que grau é efetivada a medição da segurança alimentar e

Quadro 1. Sites na Internet que veiculam fraudes e riscos de alimentos.

\begin{tabular}{|c|c|}
\hline Título & Links \\
\hline Azeite extra virgem só no rótulo & http://www.proteste.org.br/Serp/ShowResults?keyword=azeite+de+oliva \\
\hline $\begin{array}{l}\text { Dez alimentos que você consome } \\
\text { todo dia e que são falsos }\end{array}$ & $\begin{array}{l}\text { http://curiosidadeterra.blogspot.com.br/2015/03/10-alimentos-que-voce-consome-todo- } \\
\text { dia.html }\end{array}$ \\
\hline $\begin{array}{l}\text { Riscos a mesa: episódio do leite adul- } \\
\text { terado acende luz amarela na segu- } \\
\text { rança dos alimentos }\end{array}$ & $\begin{array}{l}\text { http://zerohora.clicrbs.com.br/rs/economia/noticia/2013/05/risco-a-mesa-episodio-do-leite- } \\
\text { adulterado-acende-luz-amarela-na-seguranca-dos-alimentos-4142014.html }\end{array}$ \\
\hline $\begin{array}{l}\text { Alimentos que parecem saudáveis, } \\
\text { mas não são }\end{array}$ & $\begin{array}{l}\text { http://revistacrescer.globo.com/Criancas/Alimentacao/noticia/2013/04/10-alimentos-que- } \\
\text { parecem-saudaveis-mas-nao-sao.html }\end{array}$ \\
\hline Salmão, uma carne não tão saudável & $\begin{array}{l}\text { http://www.ecycle.com.br/component/content/article/62/2929-salmao-carne-saudavel-riscos- } \\
\text { pcbs-bifenilos-policlorados-saude-humano-atividades-antropicas-fluidos-dieletricos-peixes- } \\
\text { criacao-selvagem-aquicultura-cativeiros-recomendacoes-contaminacao-enlatado-grelhado- } \\
\text { gordura.html }\end{array}$ \\
\hline
\end{tabular}


nutricional a partir de alguns indicadores já pleiteados no Plano Nacional de Segurança Alimentar e Nutricional 2012/201521. Especialmente quanto à disponibilidade de alimentos descreve-se, como exemplo, a produção anual interna de carne bovina, frango e milho para consumo humano, com agregação territorial nacional tendo como fonte primária os dados da Companhia Nacional de Abastecimento ${ }^{21}$. Esse indicador parece ser emblemático quanto à lacuna referente à análise dos impactos do processo agroindustrial de produção desses itens e quanto a falta de consenso com os parâmetros do que é um modelo de produção seguro e saudável de alimentos - nas mais diversas dimensões desses termos.

Diante desse quadro de inquietudes, evidencia-se a necessidade de superar lacunas que surgem na discussão sobre o consumo seguro de alimentos. Julga-se essencial considerar esses questionamentos e assumir a complexidade das dimensões conceituais que envolvem os conceitos de saúde e de segurança alimentar e nutricional para construir uma avaliação real dos riscos relacionados ao acesso aos alimentos.

\section{Alguns passos para a mudança}

Não se pode desconsiderar o esforço de iniciativas, especialmente oriundas das articulações presentes no espaço das instâncias de controle social da PNSAN. Um exemplo de iniciativa dessa natureza diz respeito ao estudo do Consea que demarca a mensuração do problema da (in)segurança alimentar e nutricional na concepção da produção de indicadores de monitoramento do direito humano à alimentação adequada no Brasil20. Ressalta-se que esse documento contempla sete dimensões de análise, embora ainda mantenha o desafio do encadeamento dessas informações para permitir uma leitura não fragmentada do fenômeno da insegurança alimentar e nutricional.

Pode-se considerar que o caminho de mudanças desse quadro já se iniciou também na esfera da produção do conhecimento científico. Evidencia-se essa preocupação a partir de linhas de investigação de pesquisadores ${ }^{22}$ em SAN quando seus estudos propõem a necessidade de avaliar o impacto de alimentos industrializados e contaminantes químicos sobre a saúde humana e considerar, também, a:

\begin{abstract}
... abordagem integral da Segurança Alimentar e Nutricional em programas de formação, com o objetivo de tratar questões relacionadas às diferentes conexões e processos, articuladores de uma ampla rede de organizações e movimentos sociais e de diferentes áreas de saber, existentes entre a dimensão da produção e do consumo de alimentos. Falar de Segurança Alimentar e Nutricional, vale dizer, significa inter-relacionar temas, políticas e uma rede de atores sociais $(p .114)^{22}$.
\end{abstract}

A formação de especialistas e pesquisadores em segurança alimentar e nutricional é uma iniciativa que precisa ser fomentada nos cursos de graduação e pós-graduação das diferentes áreas. Nesse sentido, o Ministério da Educação pode estabelecer marcos legais das diretrizes curriculares, bem como a inserção e evidenciação de conteúdos explícitos de SAN nos cursos de graduação que dialogam com essa temática.

Mas ainda resta a dúvida sobre quem é o agente facilitador desse conteúdo, uma vez que muitos dos especialistas, pesquisadores e docentes, sem acesso a uma formação interdisciplinar, não conseguem abarcar os princípios da segurança alimentar e nutricional e a discussão sobre a sustentabilidade na Nutrição ${ }^{18,23}$. Por esse motivo, o estudo supramencionado referente à ampla inserção de segurança alimentar e nutricional em programas de formação enfatiza a necessidade de recorrer a "um processo educativo de caráter dialógico" (p.103) ${ }^{22}$ que promova a relação entre diversos campos do conhecimento.

Outra questão importante a destacar é a necessidade de construir um consenso - ou, pelo menos, minimizar as diferenças -, no conceito de 
alimentação saudável que vigora dentro da ciência da Nutrição. Essa diferença é especialmente visível entre as áreas de Nutrição Clínica e de Nutrição Social.

A emergente proposta de medicalização da Nutrição Clínica em sua visão biomecânica e funcional do alimento como instrumento terapêutico, reduzindo-o de sua integralidade e enfatizando sua composição micronutricional, acaba por minimizar ou desconsiderar o patrimônio social que os sistemas agroalimentares tradicionais representam à construção e consolidação de um estado nutricional saudável. Cabe ressaltar que essa é uma tendência contrária à perspectiva socioambiental e cultural presente na assertiva conceitual da segurança alimentar e nutricional. Nesse sentido, tem-se questionado os padrões de abordagem dos problemas nutricionais que se caracterizam como situações de insegurança alimentar e nutricional. Como exemplo, tem-se a obesidade, tema sobre o qual levantam-se discussões quanto ao sucesso relativo da organização do cuidado assistencial, às lacunas de evidências sobre os reais fatores de risco à saúde nos sujeitos em sobrepeso, à efetividade dos resultados alcançados com medidas extremas para perda de peso - com destaque para processos cirúrgicos e intervenções dietéticas hiper restritivas que geram outros desequilíbrios à saúde -, e a sua capacidade de reverter o vínculo entre excesso nutricional e aumento da morbimortalidade ${ }^{24}$.

A tendência clínica de estimular o consumo de alimentos funcionais, light e diet é também conflitante com a preocupação da área em relação ao consumo de alimentos industrializados, altamente lucrativos para a indústria alimentar e com repercussões negativas para a saúde humana. Diante disso, percebe-se um significativo movimento de revisão conceitual que questiona a abordagem intervencionista baseada no modelo clínico e individual do início do desenvolvimento da Saúde Pública. Essa abordagem assume, gradativamente, os conceitos de segurança alimentar e nutricional e de alimentação saudável e adequada, fundamentados nas diretrizes das políticas nacionais de alimentação e nutrição. Estas, por sua vez, têm sido transpassadas pelos princípios do ideário de Promoção da Saúde e dos movimentos de Reforma Sanitária e de Saúde Coletiva.

A elaboração de delineamentos dentro da abordagem socioantropológica, histórica e geográfica, bem como a construção de reflexões e de indicadores que consideram a perspectiva cultural, já tem sido foco de alguns estudos ${ }^{25-31}$. Essas pesquisas preocupam-se com a produção do conhecimento com ênfase na cultura como elemento chave para construir indicadores de desenvolvimento humano e social. Dessa forma, podem contribuir para a construção de uma metodologia em segurança alimentar e nutricional que incorpore subjetividades nas dimensões individual e coletiva, assim como a perspectiva processual da questão alimentar e nutricional forjada na organização das sociedades.

Um importante movimento social foi deflagrado no Brasil para inserir, em 2010, o Direito Humano à Alimentação Adequada na Constituição Nacional. Considera-se que o tema deve ser estudado como eixo transversal do sistema de ensino para que o conceito se expanda e possa manter diversos canais de socialização junto aos diferentes segmentos da sociedade em seus contextos reais. Esse conceito precisa ser compreendido na ótica de sua realização cotidiana, e na perspectiva dos elementos dos diferentes setores que o forjam.

Percebe-se, na dimensão política, que a consolidação do Direito Humano à Alimentação Adequada no mais importante marco legal nacional tem potencial para gerar estratégias sociais e governamentais para o enfrentamento do processo de produção industrial dos alimentos, pressionando as indústrias desse setor, os órgãos reguladores do Estado e as instituições de direito do consumidor.

Entretanto, é importante ressaltar que exigir uma alimentação adequada e saudável vai muito além de promover a saúde do consumidor. Significa, também, interferir nas repercussões socioambientais do sistema agroalimentar dominante e fortalecer a proposta de soberania ali- 
mentar do País, não deixando de ser, estas premissas e princípios, base de um amplo conceito de saúde pública e do ideário de promoção da saúde ${ }^{4}$.

\section{CONSIDERAÇÕ ES FINAIS}

Sem desconsiderar o acúmulo de experiência sobre a produção do conhecimento na área de segurança alimentar e nutricional, bem como os avanços nas estratégias públicas para o enfrentamento da insegurança, a maior contribuição deste texto foi engatilhar uma reflexão que visa consolidar a PNSAN. Considera-se fundamental que grupos de pesquisa estejam motivados e engajados para elaborar novas metodologias de abordagem intersetorial que foquem na construção de instrumentos de coleta de informação e de indicadores de avaliação mais abrangentes e sistêmicos.

Não se ignora o desafio metodológico de abordar todos os aspectos que envolvem a segurança alimentar e nutricional de uma população em um estudo. Muitas vezes, a avaliação do estado nutricional, do consumo e da seguridade sanitária dos alimentos pode dar conta dos objetivos de um estudo - o que não é menos importante ou isento de complexidade. Porém, o reconhecimento da limitação das pesquisas para avaliar as múltiplas dimensões que envolvem a segurança alimentar e nutricional pode instigar a construção de outros indicadores e de um retrato mais legítimo do verdadeiro e ambicioso anseio em promover SAN. Em resumo, reconhecer e mencionar essa limitação deve ser uma preocupação dos estudos sobre o assunto.

Assim, considera-se que um caminho de análise dessa lacuna pode estar na reflexão sobre se e em que medida as escalas de mensuração da SAN conseguem efetivamente apontar e abarcar as complementaridades entre a vivência da insegurança alimentar e nutricional (experiência dos sujeitos que sofrem) e os processos e agentes de produção desse fenômeno. Nesse ponto é desejável incluir a leitura do processo saúde-doença e seus determinantes socioambientais e o cuidado do padecer pelas morbidades que são produto da insegurança alimentar e nutricional como campos a serem explorados em profundidade nos estudos dedicados a essa temática.

\section{COLABORADORES}

E AZEVEDO colaborou no desenho e concepção do artigo, redação e revisão crítica final do texto. MTGO RIBAS colaborou na concepção do artigo, redação crítica e revisão final do texto.

\section{REFERÊ NCIAS}

1. Nascimento RC. O papel do Consea na construção da política e do sistema nacional de segurança alimentar e nutricional [tese]. Rio de Janeiro: Universidade Federal Rural do Rio de Janeiro; 2002.

2. Brasil. Presidência da República. Decreto $n^{\circ} 7.272$, de 25 agosto de 2010. Regulamenta a Lei $n^{\circ}$ 11.346, de 15 de setembro de 2006, que cria o Sistema Nacional de Segurança Alimentar e Nutricional - SISAN com vistas a assegurar o direito humano à alimentação adequada, institui a Política Nacional de Segurança Alimentar e Nutricional PNSAN, estabelece os parâmetros para a elaboração do Plano Nacional de Segurança Alimentar e Nutricional, e dá outras providências. Diário Oficial da União. 2010.

3. Azevedo E. Riscos e controvérsias na construção social do conceito de alimento saudável: o caso da soja. Rev Saúde Pública. 2011; 45(4):781-8. http:// dx.doi.org/10.1590/S0034-89102011000400019

4. Azevedo E. O valor nutricional na construção do conceito de alimento saudável: uma discussão a partir dos alimentos orgânicos e da promoção da saúde. In: Mialhe FL, Pelicioni MCF, organizadores. Educação e promoção de saúde: teoria e prática. Rio de Janeiro: Editora Santos; 2012. p.257-69.

5. Conselho Nacional de Segurança Alimentar e Nutricional. Relatório Final. GT - alimentação adequada e saudável. Brasília: Consea; 2007 [acesso 2013 jan 14]. Disponível em: http://www.planalto. gov.br/Consea/static/documentos/Tema/ AlimentacaoAdequa/RelatorioFinal.pdf

6. Conselho Nacional de Segurança Alimentar e Nutricional. Princípios e diretrizes de uma política de 
segurança alimentar e nutricional. Anais da I Conferência Nacional de Segurança Alimentar e Nutricional. Brasília: Consea; 2004.

7. Instituto Brasileiro de Geografia e Estatística. Pesquisa Nacional por Amostra de Domicílios (PNAD). Segurança alimentar 2013. Rio de Janeiro: IBGE; 2010.

8. Pérez-Escamilla R, Segall-Corrêa AM. Food insecurity measurement and indicators. Rev Nutr. 2008; 21(Suppl.):15-26.

9. Instituto Brasileiro de Análises Sociais e Econômicas. Repercussões do Programa Bolsa Família na segurança alimentar e nutricional das famílias beneficiadas. Rio de Janeiro: Ibase; 2008 [acesso 2014 fev 20]. Disponível em: http://www5.ensp.fiocruz. br/biblioteca/dados/txt_385317780.pdf

10. Kepple AW, Gubet MB, Corrêa MAS. Instrumentos de avaliação da Segurança Alimentar e Nutricional. In: Taddei JA, Lang RMF, Longo-Silva G, Toloni MHA, organizadores. Nutrição saúde pública. São Paulo: Rubio; 2010. p.73-98.

11. Burlandy L, Salles-Costa R. Segurança alimentar e nutricional: concepções e desenhos de investigação. In: Kac G, Sichieri R, Gigante D, organizadores. Epidemiologia nutricional. Rio de Janeiro: Fiocruz; 2007. p.485-502.

12. Nascimento $A L$, Silva $F C L$, Gonçalvez $P$, Mais $S R$, Schneider S, Lira PIC. A construção de capacidades e meios de vida na garantia da segurança alimentar do rural pernambucano. Segur Aliment Nutr. 2012; 19(2):50-62.

13. Nascimento AL, Lira PIC, Maia SR. Posse da terra e produção de alimentos em domicílios com insegurança alimentar na Zona da Mata e Semiárido brasileiros. In: Gehlen VRF, Lainé PCV, organizadores. Costurando com fios invisíveis: a fragmentação do território rural. Recife: Editora Universitária da UFPE; 2012. p.361-75.

14. Sampaio MFA, Kepple AW, Segall-Corrêa AM, Oliveira JTA, Panigassi G, Maranha LK, et al. (In) Segurança alimentar: experiência de grupos focais com populações rurais do Estado de São Paulo. Segur Aliment Nutr. 2006; 13(1):64-77.

15. Carneiro FF, Pignati W, Rigotto RM, Augusto LGS, Rizollo A, Muller NM, et al. Dossiê Abrasco: um alerta sobre os impactos dos agrotóxicos na saúde. $1^{\text {a }}$ Parte. Rio de Janeiro: Abrasco; 2012.

16. Agência Nacional de Vigilância Sanitária. Relatório anual do Programa Nacional de Análise de Resíduos de Agrotóxicos em Alimento. Brasília: Anvisa; 2008 [acesso 2014 mar 23]. Disponível em: http://www. anvisa.gov.br/toxicologia/residuos/resultados_ PARA_2008.pdf
17. Domene SMA. Tendências de Consumo de alimentos industrializados pela população brasileira. In: Taddei AC, organizador. Jornadas científicas do Núcleo Interdepartamental de Segurança Alimentar 2004-2005. Barueri: Minha Editora; 2007. p.89-100.

18. Sakamoto L. Os alimentos que estão em risco de extinção no Brasil. Rio de Janeiro: Articulação Nacional de Agroecologia; 2013 [acesso 2013 nov 2]. Disponível em: http://www.agroecologia.org.br/ index.php/noticias/303-os-alimentos-que-estaoem-risco-de-extincao-no-brasil

19. Azevedo E, Rigon SA. Sistema alimentar com base no conceito de sustentabilidade In: Taddei JA, Lang RMF, Longo-Silva G, Toloni MHA, organizadores. Nutrição Saúde Pública. São Paulo: Rubio; 2010. p.543-60.

20. Conselho Nacional de Segurança Alimentar e Nutricional. A segurança alimentar e nutricional e o direito humano à alimentação adequada no Brasil. Relatório do Consea 2010. Indicadores de monitoramento da Constituição de 1988 aos dias atuais. 2010 [acesso 2011 jun 5]. Disponível em: http:// repositorio.aev.edu.br/files/ef8691fe7119b49997e 13d3be31d.pdf

21. Câmara Interministerial de Segurança Alimentar e Nutricional. Plano nacional de segurança alimentar e nutricional - 2012/2015. Brasília: Caisan; 2011.

22. Costa CA, Bogus CM. Significados e apropriações da noção de segurança alimentar e nutricional pelo segmento da sociedade civil do Conselho Nacional de Segurança Alimentar e Nutricional. Saúde Soc. 2012; 21(1):103-14. http://dx.doi.org/10.1590/s0 104-12902012000100011

23. Menezes F. Sustentabilidade alimentar: uma nova bandeira? In: Ferreira ADD, Brandenburg A, organizadores. Para pensar outra agricultura. Curitiba: UFPR; 1998.

24. Poulain JP. Sociologias da alimentação: os comedores e o espaço social alimentar. Florianópolis: Editora da UFSC; 2004.

25. Freitas MCS, Pena PGL. Segurança alimentar e nutricional: a produção do conhecimento com ênfase nos aspectos da cultura. Rev Nutr. 2007; 20(1):69-81. http://dx.doi.org/10.1590/S1415-527 32007000100008

26. Donders Y, Laaksonen A. Encontrando maneiras de medir a dimensão cultural nos direitos humanos e no desenvolvimento. Observatório Itaú Cultural. 2011; 11:89-114. http://www.itaucultural.org.br/ bcodemidias/001813.pdf

27. Azevedo E. Alimentação e modos de vida saudável. Saúde Rev. 2004; 13:31-6.

28. Freitas MCS, Pena PGL, Fontes GAV, Silva DO, Santos LAS, Mello AO, et al. Uma leitura humanista 
da nutrição. In: Freitas MCS, Fontes GAV, Oliveira $\mathrm{N}$, organizadores. Escritas e narrativas sobre alimentação e cultura. Salvador: EDUFBA; 2008. p.207-15.

29. Santos SMC. Alimentação e nutrição: um diálogo com os princípios da qualidade de vida. In: Freitas MCS, Fontes GAV, Oliveira N, organizadores. Escritas e narrativas sobre alimentação e cultura. Salvador: EDUFBA; 2008. p.261-275.

30. Ribas MTGO. A segurança alimentar e nutricional na produção da cidade: reflexões a partir do Bairro
Capão da Imbuia [mestrado]. Curitiba: Universidade Federal do Paraná; 2005.

31. Ribas MTGO. Estado nutricional e hipertensão arterial: ensaio de análise socioespacial no bairro Capão da Imbuia - Curitiba (PR). Geografias. 2006; 2(2):96-120. 
